REVIEWS

\title{
An Update on In Utero Gene Therapy for Cystic Fibrosis
}

\author{
Patrick Chua', Suzanne Zhou', Miller Richmond', Siena Romano \\ ${ }^{1}$ Georgetown University School of Medicine \\ Keywords: in utero treatment, cystic fibrosis \\ https://doi.org/10.52504/001c.16782
}

Georgetown Medical Review

Vol. 4, Issue 1, 2020

\begin{abstract}
Cystic fibrosis (CF) is an inherited, chronic disease caused by a gene mutation that leads to a malfunctioning $\mathrm{CF}$ transmembrane regulator (CFTR) protein channel in cells. The life expectancy for individuals with CF has continually increased in recent decades, but is still only around 40 years of age. Current treatment guidelines call for a focus on symptom management and complication reduction. New advances in scientific research with regard to prenatal screening, viral vectors for gene therapy, and CFTR-correcting treatments are making in utero gene therapy a possibility for the first time. In utero gene therapy would allow for an early correction of the gene mutation, preventing the subsequent complications in the development of the fetus and creating the opportunity for a cure for CF as opposed to only symptomatic treatment. In this article, we review recent developments in CF gene therapy and detail the current state of the science of CF screening as well as treatment.
\end{abstract}

\section{Introduction}

Cystic fibrosis (CF) is an inherited disease that affects multiple organ systems due to a mutation in the CF transmembrane regulator (CFTR) gene. The CFTR gene directs the synthesis of the CFTR protein, which forms a transport channel located in mucus, sweat, digestive enzymes, and saliva-producing cells. Although it has many other functions, the CFTR protein channel is primarily responsible for the proper regulation of chloride and other ions moving across the membrane of the aforementioned cells. The proper function of this channel contributes to the formation of watery, protective mucus that can be cleared by cilia in the organs. Mutations in this gene lead to mucus formation that is thick, difficult to clear from the airways, and prone to infection. ${ }^{1}$

The more prominent, debilitating effects of the CFTR gene mutation occur in the respiratory and gastrointestinal/hepatobiliary tracts. The lungs are typically subject to recurrent infections, loss of surface area due to mucus obstruction, and increased inflammation. This leads to a loss of lung function and irreversible bronchiectasis. Gastrointestinal and digestive effects include bowel obstructions, pancreatic exocrine and endocrine dysfunction, and general discomfort. Many children with CF, who are not identified during a prenatal or newborn screening, are found to have $\mathrm{CF}$ after experiencing malnutrition and failing to thrive. This is due to pancreatic insufficiency-lack of absorption of proteins, fats, and some vitamins - being one of the first symptoms of CF to manifest in a newborn. Consequently, symptoms of CF can start to affect the long-term development of children before the disease is even noticed. In fact, early nutritional supplementation has been shown to improve lung function later in life. ${ }^{2,3}$ 
Since 1938, when CF was discovered, treatment has largely focused on improving the symptoms that are caused by the defective CFTR gene, such as recurrent infections with antibiotics. This has led to an improvement in life expectancy from around 5 months when the disease was discovered to around 40 years of age today. ${ }^{3}$ There are estimated to be 33000 individuals living with the disease in the United States, and for the first time in 2014, more adults with $\mathrm{CF}$ were alive than children, marking a significant achievement in terms of longevity of those living with the disease. Individuals of all ages with CF often require intensive, expensive medical care and services to survive and improve their quality of life. Unfortunately, most patients with CF die of respiratory failure associated with a lengthy hospital stay. ${ }^{3}$ Although there has been a substantial increase in the number of years lived by people with $\mathrm{CF}$, the quality of life of these patients is highly variable, and they still have a lower life expectancy than people without CF. With recent developments in screening, gene therapy, and CFTR-correcting treatment, there is a significant opportunity to both lengthen and improve the lives of individuals with CF by applying these developments in utero. Here we review the recent developments and detail the current state of the science of CF screening and treatment.

\section{Treatment and Screening for CF}

\section{Current Treatment}

Currently, CF is primarily treated with symptom-management and complication-reduction approaches. As will be discussed further on in this article, some genotypes can be treated with CFTR-correcting treatment, but this personalized therapy is not available to all patients with CF. The primary goals for care include avoiding malnutrition through pancreatic enzyme supplementation, postponing lung disease progression, and screening and monitoring for complications of the disease. Additional goals for care focus on maintaining the quality of life and mental health for the patient as well as their caregiver. Pulmonary exacerbations are one of the main drivers of reduced quality of life, hospitalizations, and death for individuals with CF. Daily maintenance treatment for the lungs includes the use of chest vests, breathing techniques, aerosolized saline, and other devices to clear mucus from the airways. Even with adherence to these protocols, it is common for patients to require long courses of antibiotics due to the development of recurrent pneumonias caused by Pseudomonas Aeruginosa and other atypical pathogens. This has led to increasing problems with antibiotic resistance among both the general and CF population. ${ }^{2}$ Symptom-management and complicationreduction approaches have played an important role in improving the lives of patients with CF, but they also highlight the need for a focus on prevention of these CF-related issues.

Many of these treatments are labor-intensive, time-consuming, and unpleasant for patients. It is important to assess, as well as treat, anxiety and depression in patients. Studies have shown that untreated anxiety and depression in patients with CF can lead to less adherence to the medication regimen and increased 
lung complications. Intensive, life-long treatment; risk of complications; and the maintenance of quality of life are all grounds for focusing treatment on the earliest part of life as possible to increase the number of individuals who are either cured of CF or living with milder symptoms of the disease.

\section{In Utero Treatment}

In addition to reduced quality of life and short life-span, early consequences of CF, such as malnutrition and irreversible damage to cells, call for prenatal screening, genetic counseling, and future in utero treatment of the disease. Ideally, in utero gene therapy for CF would allow for a DNA molecule to be spliced, carried, and inserted into a genome that would confer the correct physiological function of the CFTR gene and subsequent protein. Additionally, it would be ideal to have high mitotic stability of the treatment, allowing for replication of the correct gene to counteract the mutations in the CFTR gene of the fetus. ${ }^{4}$ The benefits of gene therapy during this period are numerous. CFTR protein function could be improved before it can cause irreversible damage to newborn tissues and organs and treatment of a fetus is more efficient in terms of vector to cell ratio. Offering prenatal prevention of CF would allow for an option other than accepting the affected child or pursuing an abortion: empowering parents to improve the life-span of their child. ${ }^{4}$ The key to implementation of this future option in the clinic is prenatal screening and genetic counseling for CF, otherwise, application of in utero gene therapy will be limited.

\section{Screening}

$\mathrm{CF}$ is an autosomal recessive genetic disorder that many parents are unaware they are carriers for. The American College of Obstetricians and Gynecologists guidelines currently recommend that all pregnant patients, or all patients planning to become pregnant, be screened to check for carrier status. Ideally, this should be performed before pregnancy. ${ }^{5}$ There are more than 1800 identified mutations that can cause CF. Unlike other prenatal screenings, CF screening only needs to be completed one time. Because the test is based on the genetics of both parents, the outcome of the test will not change with each pregnancy. If a patient has been previously screened, CF screening results should be documented, but testing should not be repeated. If one parent is a carrier, the child will likely become a carrier too. Being a carrier would demonstrate no signs physically or clinically, and it would only be detected through genomic screening. However, if both parents are carriers, the child would have a $25 \%$ chance of having CF. Therefore, prenatal diagnosis offers many benefits to parents, such as increased time for parents to familiarize themselves with the disease and to prepare for the significant time and financial burden care will entail. ${ }^{6-8}$

In response to surveys investigating physician practices on prenatal screening of CF, $11.3 \%$ of physicians reported that they did not routinely discuss a family history of CF with either parent, $13.4 \%$ reported not providing information on 
screening for CF, and $34.2 \%$ did not offer carrier screening to every pregnant patient. ${ }^{8,9}$ Making the proper adjustments to improve those numbers reported in the surveys, in addition to patient education, would increase the probability of an early diagnosis. In addition to issues in physician adherence to screening guidelines, a study indicated that this disparity can, in part, be attributed to events outside of the physician's control, such as frequency and timing of evaluations, the age of the fetus, and parental attitudes towards genetic testing. 8,10

These parental attitudes are not without unreasonable concern because invasive genetic testing has a $0.5 \%$ to $1.0 \%$ chance of causing miscarriage. The three most common invasive screening procedures are amniocentesis, chorionic villus sampling, and fetal blood sampling. Amniocentesis is the sampling of amniotic fluid using a hollow needle inserted into the uterus. Chorionic villus sampling is the removal of tissue from the villi of the chorion, which forms the fetal part of the placenta. Fetal blood sampling is a procedure during which blood is withdrawn from the fetus during pregnancy. In a recent study, amniocentesis, chorionic villus sampling, and fetal blood sampling demonstrated miscarriage rates at $0.7 \%, 0.23 \%$, and $1.19 \%$, respectively. ${ }^{11}$ While these numbers are relatively low, of the carrier adults with CF who were surveyed, only $43.5 \%$ reported willingness to have invasive testing for $\mathrm{CF}$, and 94.4\% reported a preference for noninvasive prenatal testing, demonstrating a need for further development of noninvasive testing procedures. ${ }^{12}$

In terms of noninvasive testing, one method involves using cell-free fetal DNA (cfDNA) in maternal serum. This requires a maternal blood sample, and thus, avoids the risk of miscarriage associated with invasive diagnostic tests. ${ }^{12}$ When a woman is pregnant, her blood contains DNA fragments from the placenta that has a genetic makeup identical to that of the developing baby. Analyzing this DNA may help determine whether there is an increased or decreased risk of CF. However, cfDNA screening is not a diagnostic test; it cannot provide a definitive result. If the cfDNA results indicate an increased risk, invasive tests, such as amniocentesis, can then be performed. If the cfDNA results indicate a low risk, any additional steps would need to be discussed with the physician because low risk does not equate to no risk. Additionally, cfDNA screening results include the possibility of false-positives. CfDNA screening has no risk of pregnancy complications, such as miscarriage, and results can either provide a false sense of relief or uncertainty, if incorrectly interpreted. Furthermore, using cfDNA excludes the paternal mutation in couples carrying different CF mutations, and is a labor-intensive approach for implementation into routine clinical practice. ${ }^{12}$

Recently, next-generation sequencing assays have been developed to detect the most common CF mutations for exclusion of the paternal mutation in maternal plasma, through a polymerase chain reaction protocol. ${ }^{12}$ The limitation to this, however, is that it requires isolating paternal DNA to make 
a primer. ${ }^{8,13}$ In 2008, researchers enhanced that protocol by attaching fluorescently labeled extensions, creating fluorochrome-labeled primers. ${ }^{14}$ This technique still required designing a primer to match the allele from the paternal DNA. ${ }^{8}$ However, the detection of the allele was by a more straightforward electropherogram, eliminating the burden of electrophoresis. ${ }^{8}$ Additionally, this technique relies on the recognition of the nucleotides upstream of the single-nucleotide polymorphism of interest, not an identical match, which simplifies the amplification. ${ }^{14}$

The use of fluorochrome-labeled primers significantly reduces the cost as well as the time and handling burden associated with noninvasive screening. ${ }^{8}$ In a recent study, researchers reliably predicted mutation status in all control and maternal plasma samples using this method. ${ }^{12}$ However, a large proportion of parents reported they would have the test to prepare for the birth of a baby affected with CF, rather than to guide decisions around termination of pregnancy. Studies have analyzed the potential uptake data of noninvasive prenatal testing over invasive testing, and it is estimated to cost $£ 9025$ (US $\$ 11808$ ) for paternal mutation exclusion, and $£ 26510$ (US \$34 684) for direct diagnosis, per 100 pregnancies at risk of CF. These findings have clear consequences for the cost of prenatal testing for CF because the likely increase in uptake means that the cost of a prenatal diagnostic service will be significantly higher than the current care pathway based on invasive testing, as more tests make general prenatal services more expensive for the pregnancy. ${ }^{12}$ These potential economic implications highlight the need for further work and consideration of issues that may arise as more tests are developed. Overall, screening for CF has greatly improved in recent years, allowing more families to know their carrier status. This allows for more opportunities for treatment of CF in utero, improving the lives for patients with common CF genetic mutations. ${ }^{12}$

\section{CFTR Potentiators}

CFTR potentiators have been shown to improve the function of the CFTR channel through the restoration of the mutation in the CFTR protein that is responsible for CF. ${ }^{15}$ Under normal circumstances, the CFTR protein is an ATP-gated chloride channel located on the surface of epithelial cells that is responsible for regulating transepithelial movement of water, bicarbonate, and electrolytes into the exocrine tissues of multiple organ systems. The degree to which there are defects in chloride transport varies depending on the CFTR gene mutation, leading to differing CF disease phenotypes. While there are more than 1800 CFTR mutations, the most common gating mutation is G551D, which has been identified in $2 \%$ to $5 \%$ of patients with CF. CFTR potentiators target the underlying defect in mutated CFTR protein and have shown significant clinical benefit in patient populations with a wide range of CFTR genotypes. ${ }^{16}$ 
VX-770 (Ivacafrtor), a CFTR potentiator, has proven to be one of the most effective treatments of CF. A 2010 study showed that treatment with VX-770 improved CFTR channel activity and lung function in patients with the G551D gating mutation. ${ }^{17}$ The double-blind, placebo-controlled, multicenter study followed up 39 randomly assigned adults with CF and at least 1 G551D CFTR allele. ${ }^{17}$ After 28 days, researchers discovered that the group of patients who received $150 \mathrm{mg}$ of VX-770 had a median change in nasal potential difference of $-3.5 \mathrm{mV}(P=.13$ vs placebo $)$, a median change of the level of sweat chloride of $-59.5 \mathrm{mmol}$ per liter $(P=.02$ vs placebo), and a median change in the percentage of predicted forced expiratory volume in 1 second of $8.7 \%$ ( $P=.56$ vs placebo). ${ }^{17,18}$ Following the positive results published by $N E J M$ and multiple other studies that yielded similar findings in support of the effectiveness of VX-770 among patients with the G551D gating mutation, the drug became accepted as an established, reliable postnatal treatment option for patients with CF and the most common gating mutation.

A subsequent in vitro study in 2012 aimed to investigate the effectiveness of VX-770 with other gating mutations. Researchers used patch-clamp electrophysiology to directly measure the amount of time the CFTR channel stayed open along with clinical measures of lung function and chloride transport through CFTR. ${ }^{19}$ The study found that VX-770 was associated with an increase in the time the channel stayed open and an increase in chloride transport in patients with G551D CFTR gating mutation as well as those with non-G551D CFTR gating mutations including G178R, S549N, S549R, G551S, G970R, G1244E, S1251N, S1255P, and G1349D CFTR gating mutations. ${ }^{16,20}$ Those findings were supported in 2014 when a larger, international, multicenter clinical research study provided further evidence that VX-770 is safe and efficacious in patients with CF and non-G551D gating mutations. ${ }^{21}$ Due to its proven benefits among diverse patient populations, VX-770 continues to be one of the most widely used treatments for CF in male and female patients of all ages.

VX-770 is currently approved for patients with CF aged 1 year or older and is orally administered. However, because the development of CF disease is understood to begin in utero, studies are also evaluating the potential of VX-770 to be an effective prenatal treatment given to patients in utero. Due to the practical limitations of using human participants, a recent study generated knock-in ferrets harboring the G551D CFTR mutation allele using recombinant adeno-associated virus-mediated homologous recombination followed by somatic cell nuclear transfer. ${ }^{22}$ Pregnant jills were given $20 \mathrm{mg} /$ $\mathrm{kg}$ body weight of oral VX-770 beginning on the 28th day of gestation and researchers chose to maintain the dosage until the newborn ferrets reached 14 days old. After 14 days, the newborn ferrets received an increased dose of $10 \mathrm{mg} / \mathrm{kg}$ daily. The study found that in utero administration of VX-770 was protective against developmental pathologies in the pancreas, intestine, and male reproductive tract. Further research indicated that continuation of 
VX-770 postnatally was also correlated with better pancreatic exocrine function, glucose tolerance, growth, and survival, as well as less mucus and bacterial infections in the lung. Ceasing VX-770 administration postnatally was found to reestablish CF and this occurred most rapidly when stopping the treatment within 14 days of birth. ${ }^{22}$ Although additional research with human participants is imperative before the results can be applied to treatment protocols for patients with CF, these findings yield supportive evidence that VX-770 may be an advantageous in utero treatment option and suggests the need for funding of future research to explore its potential. ${ }^{22}$

\section{Gene Therapy}

Gene therapy has long been a topic of extensive biomedical research and debate. Gene therapy has for many years been thought of as having great potential for treating and curing genetic diseases, of which CF is of great interest and relevance. Efficient and effective delivery of a recombinant gene for therapy has been a primary obstacle in the development of gene therapy technology. Over the last few decades, a variety of methods for targeted gene therapy have been created. These methods include viral vectors, CRISPR/ Cas9, and plasmids. ${ }^{23}$

\section{Viral Vectors}

Research into the nature of viruses has created a key field of study in gene delivery for gene therapy. It is well known that viruses exert their pathogenic effects via insertion of viral genetic material into host cells, and in some cases, integration into host cell genomes. This mechanism provides opportunity for gene delivery. Three key virus families have been used for gene therapy: adenoviruses, retroviruses, and lentiviruses, a notable subtype of retroviruses.

Retroviruses, such as human T-cell lymphocytic virus, are a classic example of a virus class that infiltrate host cells and integrate genetic material within a host genome. Retroviruses offer many advantages in gene therapy, such as the ability to transform a simple single-stranded RNA genome into a doublestranded DNA molecule that integrates into host DNA in a stable manner. ${ }^{24}$ This stability allows retroviral vectors to permanently modify the genome of the host cell. ${ }^{25}$ However, many concerns exist for retroviral gene therapy. Most notably, a recent clinical trial of gene therapy for use in X-linked severe combined immunodeficiency showed promising results; however, a minority of patients in that trial later developed T-cell leukemia due to the treatment. ${ }^{26}$ Fortunately, more recent work has developed techniques for increasing the safety of retrovirus gene therapy vectors. Among these are techniques such as targeted local administration, splitting of the viral genome, vector modifications including self-inactivating vectors, and co-transfection of a suicidal gene under the control of an inducible promoter. ${ }^{27}$ These modifications have helped lead to gene therapy products using viral vectors, such as Gendicine and AdH101, with good safety profiles and limited adverse effects. ${ }^{28}$ Additionally, lentiviruses, a subtype of retroviruses, are able to infect 
cells at all stages of the cell cycle. ${ }^{29}$ This is beneficial because it can target nondividing cells such as in patients requiring long-term treatment, for example for the production of FVIII in hemophilia A. ${ }^{28}$ Additional benefits include low rates of oncogene activation and enhanced safety in delivery. ${ }^{30}$

DNA viruses, such as adenoviruses, are also a common option in viral vectormediated gene therapy. DNA viruses are known to be nonintegrating gene therapy vectors. Adenovirus, in particular, has been cited to be among the most widely used gene therapy vectors in current clinical practice. ${ }^{28,31}$ The advantages of nonintegrating gene therapy vectors include the ability to avoid insertional mutagenesis as well as position-effect variegation, thus protecting the host genome. While these are attractive advantages for the safety of gene therapy, the effects of adenovirus-mediated therapy do not appear to be longlasting. Nonintegrating vectors progressively dilute in rapidly proliferating cells and do not escape epigenetic modification of the host cell. This limits the potential for its use in many diseases. ${ }^{32} \mathrm{~A}$ study investigated the potential of adenoviruses in resolving gastrointestinal abnormalities in CF mouse models. S489X CF knockout mouse strain $c f t r^{t m 1 U n c}$ was infected with $c f t r$-containing adenoviral vector in utero. The infected mice had a permanent and complete improvement of the CF phenotype. ${ }^{33}$ The study by Larson et $\mathrm{al}^{33}$ established a foundation on which to base future studies and demonstrated that in utero adenovirus therapies could potentially provide a cure for single-gene disorders. The lasting improvement of the CF model through the use of gene therapy in utero is encouraging because it is an improvement over the transient nature of postnatal gene therapy treatments.

\section{Nonviral DNA Vectors}

The use of plasmids is one of the oldest gene therapy techniques and has been studied as such since the 1990s. Plasmids are both less expensive and more stable to produce and store, making them easier to work with as gene therapy solutions. ${ }^{34}$ Plasmids rarely integrate into genomes and may be repeatedly delivered while viral vectors cannot. The plasmid safety profile has allowed for a quicker transition into clinical trials, as opposed to viral vector therapy. Clinical trials of plasmid-based gene therapy exist in phase 1 or 2 for cystic fibrosis, cancer, diabetes, heart disease, hepatitis B, hepatitis C, HIV, human papillomavirus, influenza, and malaria. ${ }^{35,36}$ Like any delivery mechanism for gene therapy, plasmids have their downsides. Gene therapy effects delivered by plasmids are often transient, with effects diminishing after every cell replication. In addition, plasmids are an inefficient method of actually delivering the gene product due to low cell uptake rates of the plasmid. ${ }^{34}$

Recent developments in CRISPR/Cas9 technology have many applications in both the research and clinical arenas. CRISPR/Cas9, which was originally borrowed from a mechanism observed in bacteria, is very efficient and easily targets specific areas of the genome, depending on the single-guide RNA. ${ }^{37}$ When compared with other gene-editing techniques, CRISPR/Cas9 has 
improved efficacy, speed, and simplicity. ${ }^{38}$ CRISPR/Cas9 has led to many medical therapy research trials since its introduction in 2013. It is currently proving to be effective in repairing CFTR function in CF organoids as well as decreasing HIV viral expression, treating hepatitis $\mathrm{B}$, and treating human papillomavirus. ${ }^{39}$ However, there are still issues with CRISPR/Cas9 that need to be ameliorated. Because CRISPR/Cas9 is a homology-directed repair system, there are occasional insertions or deletions of bases. As a result of these unintentional mutations, patients taking drugs that rely on an intact genome may no longer appropriately respond to their current treatment regimen. Without an effective alternative in place, this could prove devastating for the patient. ${ }^{40}$ Still, as an economic and effective method of delivering gene therapy, CRISPR/Cas9 may play an important role in in utero delivery of gene therapy in the future. In addition to these novel basic science research innovations using a variety of gene therapy vectors, there are currently experiments focusing on expanding the scope of current CF treatments.

\section{Conclusion}

Scientific advancement, particularly the development of novel antibiotics, has improved the quality of life and life-span of individuals living with CF, and there will be ongoing development in these areas that treat the symptoms of malfunctioning CFTR proteins. However, the hope of curing CF lies in the field of in utero gene therapy. Viral vectors equipped to insert themselves into the DNA of a developing fetus should soon be able to correct the mutations in the CFTR gene. These improvements are creating the opportunity to capitalize on early intervention in growing fetuses, thus preventing the long-term complications of defective CFTR proteins and a lack of proper transport of chloride ions. Overall progress in animal models for $\mathrm{CF}$, viral vectors, and CFTR potentiators is opening the path toward a cure.

Scientific, societal, and institutional barriers to a cure continue to exist. The heterogeneous nature of CF genetic mutations means that a cure for CF will not be one-size-fits-all; instead, gene therapy will be more individualized to meet the needs of the diverse CF patient population possessing varying gene mutations. Prenatal genetic screening is currently costly and rarely completed. Genetic counselors, who guide both genetic screening and ethical conversations about the risks and benefits of gene therapy, are chronically underfunded and underappreciated. Inequity in health care access means that too often people with chronic diseases such as CF do not have the resources required to attend their numerous medical appointments every month or receive the medications and equipment that they need. Governmental and institutional investments need to be made in these areas to reap the full benefits of progress in gene therapy research. Medical advancements have continually improved the lives of individuals with CF, and a multifaceted approach to in utero gene therapy will enable the full potential of a cure to be realized. 


\section{REFERENCES}

1. National Institutes of Health. Genetics Home Reference. https://ghr.nlm.nih.gov/. Accessed April 4, 2020.

2. Farrell PM, Kosorok MR, Laxova A, et al. Nutritional Benefits of Neonatal Screening for Cystic Fibrosis. NEngl J Med. 1997;337(14):963-969. doi:10.1056/nejm199710023371403

3. Sanders DB, Fink AK. Background and Epidemiology. Pediatr Clin North Am. 2016;63(4):567-584. doi:10.1016/j.pcl.2016.04.001

4. Conese M, Ascenzioni F, Boyd AC, et al. Gene and cell therapy for cystic fibrosis: From bench to bedside. J Cyst Fibros. 2011;10:S114-S128. doi:10.1016/s1569-1993(11)60017-9

5. Rink B, Romero S, Biggio JR Jr, Saller DN Jr, Giardine R. Committee opinion No. 691: obesity in pregnancy. Obstet Gynecol. 2017;121(1):213-217. doi:10.1097/01.aog.0000425667.10377.60

6. Rosenberg MA, Farrell PM. Assessing the cost of cystic fibrosis diagnosis and treatment. $J$ Pediatr. 2005;147(3):S101-S105. doi:10.1016/j.jpeds.2005.08.018

7. Van Gool K, Norman R, Delatycki MB, Hall J, Massie J. Understanding the Costs of Care for Cystic Fibrosis: An Analysis by Age and Health State. Value in Health. 2013;16(2):345-355. doi:10.1016/i.jval.2012.12.003

8. Nishida K, Smith Z, Rana D, Palmer J, Gallicano GI. Cystic fibrosis: A look into the future of prenatal screening and therapy. Birth Defects Res C Embryo Today. 2015;105(1):73-80. doi:10.1002/bdrc.21091

9. Morgan MA, Driscoll DA, Mennuti MT, Schulkin J. Practice patterns of obstetriciangynecologists regarding preconception and prenatal screening for cystic fibrosis. Genet Med. 2004;6(5):450-455. doi:10.1097/01.gim.0000139509.04177.4b

10. Darcy D, Tian L, Taylor J, Schrijver I. Cystic fibrosis carrier screening in obstetric clinical practice: Knowledge, practices, and barriers, a decade after publication of screening guidelines. Genet Test Mol Biomarkers. 2011;15(7-8):517-523. doi:10.1089/gtmb.2010.0228

11. Nanal R, Kyle P, Soothill PW. A classification of pregnancy losses after invasive prenatal diagnostic procedures: An approach to allow comparison of units with a different case mix. Prenat Diagn. 2003;23(6):488-492. doi:10.1002/pd.623

12. Hill M, Twiss P, Verhoef TI, et al. Non-invasive prenatal diagnosis for cystic fibrosis: Detection of paternal mutations, exploration of patient preferences and cost analysis. Prenat Diagn. 2015;35(10):950-958. doi:10.1002/pd.4585

13. Nasis O, Thompson S, Hong T, et al. Improvement in sensitivity of allele-specific PCR facilitates reliable noninvasive prenatal detection of cystic fibrosis. Clinical Chemistry. 2004;50(4):694-701. doi:10.1373/clinchem.2003.025981

14. Bustamante-Aragones A, Perlado-Marina S, Trujillo-Tiebas MJ, et al. Non-invasive prenatal diagnosis in the management of preimplantation genetic diagnosis pregnancies. J Clin Med. 2014;3(3):913-922. doi:10.3390/jcm3030913

15. Hanrahan JW, Matthes E, Carlile G, Thomas DY. Corrector combination therapies for F508del-CFTR. Curr Opin Pharmacol. 2017;34:105-111. doi:10.1016/j.coph.2017.09.016

16. Yu H, Burton B, Huang C-J, et al. Ivacaftor potentiation of multiple CFTR channels with gating mutations. Journal of Cystic Fibrosis. 2012;11(3):237-245. doi:10.1016/j.jcf.2011.12.005 
17. Accurso FJ, Rowe SM, Clancy JP, et al. Effect of VX-770 in persons with cystic fibrosis and the G551D-CFTR mutation. N Engl J Med. 2010;363(21):1991-2003. doi:10.1056/nejmoa0909825

18. Honeybourne D. Cystic fibrosis papers of the year 2010-2011. J R Soc Med. 2012;105(suppl 2):S30-S35. doi:10.1258/jrsm.2012.12s006

19. Yu H, Burton B, Huang C-J, et al. Ivacaftor potentiation of multiple CFTR channels with gating mutations. J Cyst Fibros. 2012;11(3):237-245. doi:10.1016/j.jcf.2011.12.005

20. Angelbello AJ, Chen JL, Childs-Disney JL, Zhang P, Wang Z-F, Disney MD. Using genome sequence to enable the design of medicines and chemical probes. Chem Rev.

2018;118(4):1599-1663. doi:10.1021/acs.chemrev.7b00504

21. De Boeck K, Munck A, Walker S, et al. Efficacy and safety of ivacaftor in patients with cystic fibrosis and a non-G551D gating mutation. J Cyst Fibros. 2014;13(6):674-680. doi:10.1016/ j.jcf.2014.09.005

22. Sun X, Yi Y, Yan Z, et al. In utero and postnatal VX-770 administration rescues multiorgan disease in a ferret model of cystic fibrosis. Sci Transl Med. 2019;11(485):eaau7531. doi:10.1126/ scitranslmed.aau7531

23. Cooney AL, McCray PB Jr, Sinn PL. Cystic fibrosis gene therapy: Looking back, looking forward. Genes (Basel). 2018;9(11):538. doi:10.3390/genes9110538

24. McBurney SP, Young KR, Nwaigwe CI, Soloff AC, Cole KS, Ross TM. Lentivirus-like particles without reverse transcriptase elicit efficient immune responses. Curr HIV Res. 2006;4(4):475-484. doi:10.2174/157016206778560018

25. Anson DS. The use of retroviral vectors for gene therapy-what are the risks? a review of retroviral pathogenesis and its relevance to retroviral vector-mediated gene delivery. Genet Vaccines Ther. 2004;2(1):9. doi:10.1186/1479-0556-2-9

26. Cavazzana M, Six E, Lagresle-Peyrou C, André-Schmutz I, Hacein-Bey-Abina S. Gene therapy for X-linked severe combined immunodeficiency: Where do we stand? Hum Gene Ther. 2016;27(2):108-116. doi:10.1089/hum.2015.137

27. Yi Y, Noh MJ, Lee KH. Current advances in retroviral gene therapy. CGT. 2011;11(3):218-228. doi:10.2174/156652311795684740

28. Lundstrom K. Viral vectors in gene therapy. Diseases. 2018;6(2):42. doi:10.3390/ diseases 6020042

29. Chang LJ, Gay EE. The molecular genetics of lentiviral vectors: current and future perspectives. Curr Gene Ther. 2001;1(3):237-251. doi:10.2174/1566523013348634

30. Gándara C, Affleck V, Stoll EA. Manufacture of third-generation lentivirus for preclinical use, with process development considerations for translation to good manufacturing practice. Hum Gene Ther Methods. 2018;29(1):1-15. doi:10.1089/hgtb.2017.098

31. Zhu YP. Prostaglandin E2 receptor and uterine bleeding caused by intrauterine devices [in Chinese]. Zhonghua Fu Chan Ke Za Zhi. 1987;22(4):226-228, 254.

32. Wold WSM, Toth K. Adenovirus vectors for gene therapy, vaccination and cancer gene therapy. Curr Gene Ther. 2013;13(6):421-433. doi:10.2174/1566523213666131125095046

33. Larson JE, Morrow SL, Happel L, Sharp JF, Cohen JC. Reversal of cystic fibrosis phenotype in mice by gene therapy in utero. Lancet. 1997;349(9052):619-620. doi:10.1016/ $\underline{\mathrm{s} 0140-6736(05) 61567-\mathrm{x}}$ 
34. Wolff JA, Malone RW, Williams P, et al. Direct gene transfer into mouse muscle in vivo. Science. 1990;247(4949):1465-1468. doi:10.1126/science.1690918

35. Hardee CL, Arévalo-Soliz LM, Hornstein BD, Zechiedrich L. Advances in non-viral DNA vectors for gene therapy. Genes (Basel). 2017;8(2):65. doi:10.3390/genes8020065

36. Izsvák Z, Chuah MKL, Vandendriessche T, Ivics Z. Efficient stable gene transfer into human cells by the Sleeping Beauty transposon vectors. Methods. 2009;49(3):287-297. doi:10.1016/ j.ymeth.2009.07.001

37. Konermann S, Brigham MD, Trevino AE, et al. Genome-scale transcriptional activation by an engineered CRISPR-Cas9 complex. Nature. 2015;517(7536):583-588. doi:10.1038/nature14136

38. Rodríguez-Rodríguez DR, Ramírez-Solís R, Garza-Elizondo MA, Garza-Rodríguez ML, Barrera-Saldaña HA. Genome editing: A perspective on the application of CRISPR/Cas9 to study human diseases (Review). Int J Mol Med.2019;43(4):1559-1574. doi:10.3892/ijmm.2019.4112

39. Schwank G, Koo B-K, Sasselli V, et al. Functional repair of CFTR by CRISPR/Cas9 in intestinal stem cell organoids of cystic fibrosis patients. Cell Stem Cell. 2013;13(6):653-658. doi:10.1016/j.stem.2013.11.002

40. Lino CA, Harper JC, Carney JP, Timlin JA. Delivering CRISPR: A review of the challenges and approaches. Drug Deliv. 2018;25(1):1234-1257. doi:10.1080/10717544.2018.1474964 